\title{
LA POSMODERNIDAD Y LA AUTENTICIDAD PATRIMONIAL EN SU RELACIÓN CON LA CULTURA CAMPESINA Y LA EDUCACIÓN MEDIA EN CUBA Y BRASIL.
}

\begin{abstract}
POSMODERNITY AND HERITAGE AUTHENTICITY IN ITS RELATIONSHIP WITH THE CULTURE OF CAMPESINA AND MEDIA EDUCATION IN CUBA AND BRAZIL.
\end{abstract} DOI: http://dx.doi.org/10.9771/gmed.v11i1.30569

\author{
Luiz Bezerra Neto ${ }^{1}$ \\ Juan Carlos Rodriguez Cruz ${ }^{2}$
}

Resumo: O trabalho aborda a categoria do patrimônio cultural e as implicações negativas que a pós-modernidade implica em meio a um processo crescente de globalização. Uma análise do patrimônio cultural material e espiritual é realizada em concomitância com a autenticidade, com uma projeção antropológica em direção ao intangível, sem esquecer a correlação existente entre ambos os aspectos patrimoniais. Os aspectos espirituais ou intangíveis da herança cultural camponesa são valorizados, como parte da identidade cultural camponesa, que representa a base fundamental sobre a qual se baseia a identidade nacional, e o impacto que ela tem na educação média em Cuba e no Brasil e seus efeitos opostos.

Palavras-chave: pós-modernidade, patrimônio cultural, autenticidade, identidade camponesa, educação.

Resumen: El trabajo aborda la categoría patrimonio cultural y las implicaciones negativas que le acarrea la posmodernidad en medio de un proceso creciente de globalización. Se realiza un análisis del patrimonio cultural material y espiritual en concomitancia con la autenticidad con una proyección antropológica hacia lo intangible, sin dejar de tener presente la correlación que existe entre ambas vertientes patrimoniales. Se valora lo espiritual o intangible del patrimonio cultural campesino, como parte de la identidad cultural campesina, la cual representa la base fundamental en que se sustenta la identidad nacional, así como se valora el impacto que este tiene en la educación media en Cuba y Brasil y sus efectos contrarios.

Palabras claves: posmodernidad, patrimonio cultural, autenticidad, identidad campesina, educación.

Abstract: The work addresses the category of cultural heritage and the negative implications that postmodernity entails in the midst of a growing process of globalization. An analysis of the material and spiritual cultural heritage is carried out in concomitance with authenticity with an anthropological projection towards the intangible, without forgetting the correlation that exists between both heritage aspects. The spiritual or intangible aspects of the peasant cultural heritage are valued, as part of the peasant cultural identity, which represents the fundamental basis on which the national identity is based, as well as the impact it has on middle education in Cuba and Brazil and its opposite effects.

Key words: postmodernity, cultural heritage, authenticity, peasant identity, education.

Con el inicio de la posmodernidad como proceso irreversible que acarrea la pérdida de la memoria histórica, comienza la pérdida paulatina de los valores patrimoniales. Estos valores comienzan a descender en la escala axiológica y se van generalizando en todas las esferas de la vida social. Estos cambios afectan a la noción histórica, sociocultural, política, territorial y cognitiva que incluye la propia intuición sobre la 
objetividad. Estos cambios son, comentando a Lamas, concomitantes con el proceso de globalización. (LAMAS, 2013).

El patrimonio cultural tanto material como espiritual es un legado histórico. Es resultado del esfuerzo de diferentes culturas por crear y conservar su propia identidad y, al mismo tiempo, estas identidades le van dando sentido a su existencia. Entiéndase por patrimonio cultural la herencia de la cultura “...las obras de arquitectos, músicos, escritores y sabios, así como las creaciones anónimas, surgidas del alma popular, y el conjunto de valores que dan sentido a la vida, es decir, las obras materiales y no materiales que expresan la creatividad de ese pueblo; la lengua, los ritos, las creencias, los lugares y monumentos históricos, la literatura, las obras de arte y los archivos y bibliotecas. (UNESCO, 1982, p.3).

Cuando la UNESCO reconoce también como patrimonio las obras surgidas del alma popular y todo el conjunto de valores que dan sentido a la vida, está reconociendo a la tradición como parte de ese patrimonio cultural. Las tradición cultural no es la costumbre de hacer lo repetido, es pensar y sentir en lo que se trasmite, es parte intríseca del patrimonio cultural, consustancial como expresión y, por tanto, al trasmitirse tiene una connotación educativa. Esta educación se inicia en la vida familiar, y tuviera continuidad en las instituciones, si las políticas educacionales la contemplaran en sus programas currículares. Conservar la tradición o las tradiciones culturales, como parte del patrimonio cultural, desde lo socio-educativo, es defender la esencia identitaria de una colectividad social como su principal razón de ser, en un mundo en el que la posmodernidad acarrea el desarraigo patrimonial.

El destacado investigador Lamas entre sus análisis sobre la posmodernidad y el patrimonio cultural señala que “...el escenario de la posmodernidad abre una gran oportunidad para el patrimonio, porque como nos dice Marta Arjona, el patrimonio alimenta siempre en el ser humano una sensación reconfortante de continuidad en el tiempo y de identificación con una determinada tradición." (LAMAS, 2013, p.5).

Estos investigadores que suscriben no concuerdan con la interpretación de Lamas, (2013). La cita que referencia, de la destacada investigadora cubana Marta Arjona, con la cual estos autores sí coinciden, no significa que la posmodernidad le abra las puertas al patrimonio. Muchas directrices gubernamentales se implementan en concomitancia con la UNESCO para conservar el patrimonio cultural tangible e intangible, pero el desarraigo que trae consigo lo posmoderno cierra sus grandes oportunidades y desvaloriza lo creado por el hombre a lo largo de la histora. De ahí nace el esfuerzo de múltiples instituciones por atesorar estos valores que constituyen bienes culturales.

Los valores culturales se convierten, por su esencia patrimonial, en un bien cultural. Se asume como bien cultural "aquello que es físicamente parte de él y de su entorno. Un bien histórico, producto del pasado que ha sufrido cambios o deterioros ocasionados tanto por el desgaste natural como por su uso funcional (...) La suma de esos cambios se convierte de por sí en parte de su carácter histórico y de su material esencial y representa el valor intrínseco del bien; es el soporte de los testimonios históricos y de los valores culturales asociados, tanto del pasado como del presente." (DIAZ, 2009, p.6).

Se concuerda con el planteamiento anterior en que los valores patrimoniales no pueden conservarse de forma tal que no sufran cambios en el tiempo del pasado al presente. Estos son acosados 
por la dialéctica de la transformación, por tanto, lo más importante es conservar su esencia, que su esencia transcurra en el tiempo como un testimonio vivo. Y en esa esencia está la originalidad, la autenticidad, y no en los materiales con los cuales se contruyan. Razón por la cual González señala que “...son más auténticos un muro de carga o una bóveda que trabajen tal y como fue previsto originalmente, aunque todos sus ladrillos, mampuestos o dovelas sean nuevos, que un muro o una bóveda cuyos elementos hayan sido materialmente conservados pero que hayan perdido su capacidad mecánica.” (GONZÁLEZ, 1997, p. 22).

Y es que parte de sus valores se perderán en el proceso o serán reemplazados por otros. Llevarán la huella de un tiempo distinto, el de sus nuevos constructores. Se está consciente de la distancia que media entre el original y su réplica, no porque la calidad de su ejecución vaya a ser menor, o porque no sea posible saber cómo estaban resueltos todos los detalles de la obra, sino "porque toda réplica es, sin duda, una reinterpretación.” (SOLÁ-MORALES, 1993, p. 39). Razón por la cual el concepto autenticidad continúa siendo polémico a la hora de valorarlo. Este concepto se interpreta y reinterpreta de acuerdo a la concepción patrimonial que tenga cada generación.

El cocepto autenticidad ha sido abordado por la UNESCO y por múltiples investigadores como García, (2012), Sánchez, (2013), González (1997), Solá (1993), Rico, (2016), los cuales coinciden en la búsqueda de la originalidad del bien patrimonial, en su forma más que en su contenido; y no como defienden estos investigadores que suscriben, que buscan la autenticidad en la temporalidad de su esencia valoral, en constante proceso de evolución.

En el V Encuentro Regional del ICOMOS, desarrollado en Brasilia, en diciembre de 1995, se elaboró la Carta de Brasilia que plantea el tema de la autenticidad e identidad. En esta Carta se plantea que “...el significado de la palabra autenticidad está íntimamente ligado a la idea de verdad; es auténtico aquello que es verdadero, que se da por cierto, que no ofrece dudas. Los edificios y sitios son objetos materiales, portadores de un mensaje o argumento cuya validez, en el marco de un contexto social y cultural determinado y de su comprensión y aceptación por parte de la comunidad, los convierte en patrimonio." (CARTA DE BRASILIA, 1995, p.3).

Asimismo en el documento de Nara sobre Autenticidad, (1994, p.1), el comité de experto señala en el prólogo: “....reconocemos el valor del marco de discusión propuesto por el Comité del Patrimonio Mundial en su deseo de que el concepto de autenticidad garantice total respeto por los valores sociales y culturales de todos los pueblos cuando se analicen los antecedentes de los bienes que le sean propuestos para su declaratoria."

Se coincide con la UNESCO en que la autenticidad está íntimamente ligada a la idea de verdad y, con el documento de Nara, en el total respeto por los valores sociales y culturales de todos los pueblos. Pero la búsqueda de la verdad, a juicio de estos autores, se considera como el respeto y búsqueda de la condición 0 atributo de los valores originales y no del bien cultural original como tal, pues el bien cultural está expuesto a la prueba del tiempo.

El valor cultural original queramos o no cambia y en esa permutación, como proceso, lo más importante es la permanencia de su esencia en la crisis transformacional, que exprese en cualquier tiempo lo que fue y es. Esto sucede fundamentalmente con el patrimonio intangible cuando se protege de las 
penetraciones culturales y, aunque nunca está exento de ellas, la sustancia valoral en esa protección persiste, aunque cambien sus formas de expresión temporal, y emerjan nuevos elementos producto a sus interrelaciones de las cuales se encarga la dialéctica del cambio. Al respecto Koichiro Matssura quien fuera director de la UNESCO considera que “...el patrimonio inmaterial no es solamente sede de la memoria de la cultura de ayer, sino también el laboratorio donde se inventa el mañana." (CARRERA, 2018, p.1). Por tanto los valores patrimoniales no permanecen estáticos, sino en proceso de relativización, pero en esa relativización la esencia es la que no debe disiparse.

La autenticidad, como concepto, es polémico y contradictorio a la hora de valorarlo y definirlo. El patrimonio cultural lo mismo tangible que intangible sufre transformaciones y solo trasmite su razón de ser si este es protegido. Cuando a un bien cultural material se le aplica la acción del hombre o se restaura, pierde autenticidad, porque la restauración es un acto de innovación, una acción agresiva al bien cultural, por muy minuciosa que esta restauración sea en la búsqueda de la verdad, como plantea la UNESCO.

La polemicidad del concepto autenticidad señala García, (2009) se da por la propia ampliación del concepto de Patrimonio Cultural que ha conllevado necesariamente a revisar el concepto de autenticidad, término que sigue sin estar demasiado claro. Considera que la creciente diversidad de las categorías de patrimonio, de sus materiales y de los métodos de construcción o de planificación, han hecho que se acepte la idea de que debe haber normas variables para una conservación auténtica, y que hay que tener en cuenta las diferentes interpretaciones del concepto autenticidad, según las diversas tradiciones culturales que constituyen parte del patrimonio cultural.

Según la lógica de este trabajo se formula una pregunta, ¿Cómo se presenta la defensa del patrimonio cultural en los contextos de Cuba y Brasil?

En el contexto cubano existe una defensa del patrimonio cultural y de su esencia como legado del pasado. Es preocupación del Gobierno y del Estado en Cuba defender su patrimonio y su identidad, teniendo como premisa fundamental que la identidad se expresa a través del patrimonio y el patrimonio es la huella y manifestación más concreta de la identidad; así como la defensa de su patrimonio es una forma de defender la identidad nacional. ¿Pero qué dice sobre el patrimonio cultural cubano una voz autorizada desde el extranjero?

La situation de ce pays est tout à fait exceptionnelle, d'abord par rapport au reste de la Caraibe, puisqu'il détient à lui seul sept des quinze sites, culturels ou naturels, distingués par l'UNESCO pour cette région du globe [...], mais aussi par la variété de ce qui a été jugé digne d'entrer dans la mémoire collective de la planète. Aux sept sites architecturaux retenus (la vieille ville de La Havane et ses fortifications, la citadelle de Santiago, les vestiges des caféières de la région de Santiago, Trinidad et la Vallée sucrière de Los Ingenios, la vallée de Viñales, les cœurs anciens de Cienfuegos et Camagüey...) s'ajoutent en effet deux biens "naturels" (les parcs de Desembarco de Granma et d'A. Humboldt), et plus remarquable, car il s'agit du seul exemple dans la Caraibe pour le patrimoine immatériel, la labellisation de la Tumba francesa de Santiago, association de chants et de danses afro-cubains. (BÉGOT 2011, p.74). ${ }^{3}$

Repecto al patrimonio cultural en Brasil, ¿qué dice también una voz acreditada, pero en este caso de una brasileña, Guiomar de Grammont 1998? (apud, FUNARI, 2001, p.3) Esta escritora, editora, dramaturga y filósofa describe la situación de la siguiente forma: 
A distância entre as autoridades e o povo é a mesma daquela entre a sociedade civil e o passado, devido à falta de informação, ainda que os habitantes das cidades coloniais dependam do turismo para sua própria sobrevivência. Quem são os maiores inimigos da preservação dessas cidades coloniais? Em primeiro lugar, a própria administração municipal, não afetada pelos problemas sociais e ignorante das questões culturais em geral mas, às vezes, os moradores também, inconscientes da importância dos monumentos, contribuem para a deformação do quadro urbano. Novas janelas, antenas parabólicas, garagens, telhados e casas inteiras bastam para transformar uma cidade colonial em uma cidade moderna, uma mera sombra de uma antiga cidade colonial, como é o caso de tantas delas. ${ }^{4}$

Ambos criterios son diferentes respecto a la defensa de los dos patrimonios culturales. Si en Cuba las autoridades competentes preservan los bienes de la cultura y como dice Bégot, que en todo el Caribe, Cuba posee por sí solo siete de los quince sitios, culturales o naturales, distinguidos por la UNESCO para esta región del globo; en el contexto brasileño, a juicio de la destacada intelectual Guiomar de Grammont, existe ignorancia en el pueblo y un abandono de las administraciones municipales por la defensa de su patrimonio lo que acarrea consigo una pérdida de sus valores culturales, de su historia, sus tradiciones y de su propia identidad cultural.

El patrimonio cultural tangible e intangible en el contexto de este trabajo se analizará en sus múltilples relaciones dialécticas. Aunque estos autores se centrarán en el patrimonio intangible o espiritual y, desde esta vertiente, se dará una mirada al patrimonio material o tangible.

Respecto al patrimonio intangible o espiritual este ha sido trabajado por una variada autoría, entre los que ameritan ser mencionados, en primer lugar a la UNESCO y su equipo de expertos. En segundo lugar autores de la talla de Rocabado, (2004), Rosón, (2018), Velasco, (2012), Argaillot, (2013) y Tapia, (2012); los cuales han trajado de una forma u otra el patrimonio intangible desde diferentes vertientes y han realizado aportaciones a la teoría.

Según la UNESCO, en la reunión celebrada en Turín, (2001), considera que el patrimonio cultural inmaterial, son:

los procesos adquiridos por las personas tales como el conocimiento, las habilidades y la creatividad que les son heredados y desarrollados, los productos que crean y los recursos, espacios y otras dimensiones del contexto social y natural necesarias para su sustentabilidad; procesos que proporcionan a las comunidades vivientes un sentimiento de continuidad con las generaciones antecesoras y son importantes para la identidad cultural, así como para la salvaguarda de la diversidad cultural y de la creatividad de la humanidad. (Citado por DESANTES REAL, 2013, p.12).

Según los Lineamientos para la Identificación y Manejo del Patrimonio Cultural Inmaterial (LIMCI):

es una categoría que agrupa un conjunto de manifestaciones culturales que las personas consideran importantes y a las que asignan un valor especial. Estas manifestaciones tienen una amplia proyección en el tiempo y se mantienen activas al evidenciar las relaciones que los grupos humanos establecen con su entorno social, ambiental e histórico. (LIMCI, 2014, p.21).

En esta misma línea de análisis que realiza la UNESCO y presenta los Lineamientos para la Identificación y Manejo del Patrimonio Cultural Inmaterial, Carrión considera que el patrimonio cultural inmaterial:

es un Patrimonio Cultural de grupos, de comunidades o en uso por parte de individuos pertenecientes a colectividades que habitan en áreas culturales determinadas, caracterizadas por estilos de vida o de organización propios. Por tanto, además de 
constituir una argamasa que unifica a colectivos a lo largo de la Historia, cumple también funciones de adaptación al medio, de organización socio-familiar, de producción económica, de intercambio de bienes, pero también de expresión de significados que se refuerzan en marcos colectivos consensuados. (CARRIÓN, 2015, p.8)

Las definiciones anteriores se interrelacionan y se complementan y tienen como transversal la cultura. Según los criterios anteriores la cultura es la que le da sentido al patrimonio y a la identidad en sus múltiples relaciones sociales, económicas, ambientales y de diversidad en las diferentes áreas o espacios para la creación.

A partir de lo anteriormente expuesto, estos investigadores que suscriben definen al patrimonio intangible como el conjunto de conocimientos, costumbres, tradiciones, leyendas y mitos, que se han trasmitido de forma oral o escrita de generación a generación. Dicho patrimonio es la conjunción de los aportes de las culturas primigenias y de lo nuevo que se ha ido creando a lo largo de los siglos. El patrimonio intangible es el alma de la comunidad, el sentir y el comprender del pueblo a lo largo de generaciones.

El patrimonio intangible contiene al patrimonio cultural campesino y, a su vez, el patrimonio cultural campesino es parte intrínseca de la identidad cultural campesina por ser este su acervo fundamental. En el contexto de este trabajo estos autores entienden por identidad cultural campesina los valores culturales, materiales y espirituales relacionados con el medio rural, referente a las formas de vida que les sirven de contexto, heredadas y enriquecidas por sucesivas generaciones y, al mismo tiempo, se entiende por patrimonio campesino el paisaje con valor estético o histórico, construcciones, instrumentos de trabajo y otros objetos utilitarios, creencias, costumbres, tradiciones, saberes y variante idiomática, que reflejan la herencia histórico-cultural y su adaptación al contexto rural.

Por tanto, el patrimonio cultural campesino tangible e intangible, existe en una relación dialéctica, en el que la cultura espiritual y el imaginario socio-histórico permiten la subsistencia de lo físico o material. $\mathrm{Al}$ desarraigarse esa cultura espiritual o desaparecer, se desarraigan o desaparecen sus formas concretas.

El patrimonio campesino existe, en primer lugar porque existe un grupo humano que lo sustenta en sus prácticas y, en segundo lugar, por constituir ese campesinado una fuerte cultura, una cultura milenaria que llega a América en su versión mediterránea. Aun cuando es una cultura milenaria no se sugiere en modo alguno que esta cultura esté exenta de peligros con los nuevos encontronazos con este nuevo proceso civilizatorio posmoderno.

En los momentos actuales con el desarrollo de las nuevas tecnologías de la información y el éxodo a gran escala del campo a la ciudad, el peligro fundamental que corre el patrimonio cultural campesino radica en la depreciación que hacen de éste la población y las instituciones educativas; esto acarrea un proceso de desvalorización y desarraigo en las nuevas generaciones, lo que restringe su sustentación.

\section{¿Cómo se presenta este proceso en el estudiantado secundario y en sentido general en la educación media cubana y brasileña?}

En las investigaciones realizadas por estos autores respecto a la educación patrimonial campesina en la enseñanza media de Cuba y Brasil, se pudieron detectar múltiples insuficiencias que desvalorizan este patrimonio campesino que continua siendo en ambos contextos el pilar en el que se sustenta su cultura e 
identidad nacional. Ambos países son latinoamericanos, tercermundistas, con una historia de luchas emancipadoras y, aunque sus lenguas son diferentes, ambas lenguas son neo latinas, reconocidas por los lingüistas como lenguas hermanas.

Respecto a la educación en Cuba los estudiantes secundarios:

- Tienden a negar sus orígenes, costumbres y tradiciones y a asimilar rápidamente lo que ellos consideran modos de actuar más modernos; desestiman las tradiciones, la música y los bailes campesinos; la población está sujeta a un fuerte proceso de emigración hacia la ciudad, lo que conspira contra la sostenibilidad del rico patrimonio campesino;

- Muchos estudiantes perciben las tradiciones culturales de su ámbito rural como algo pasado de moda, desconociendo el valor identitario y patrimonial de dicho sistema de tradiciones, costumbres y leyendas;

- La secundaria básica rural, y la semiurbana enclavada en pequeños poblados rodeada de una población campesina, están haciendo muy poco por vincularse culturalmente con dicho medio, aun cuando gran número de estudiantes no se sienten identificados con sus raíces culturales de origen campesino, hecho que está relacionado con la despoblación de los campos y sobre todo con el creciente desarraigo;

- Los estudiantes al llegar a la escuela desvalorizan la literatura oral tradicionalmente aprendida y la secundaria básica hace poco esfuerzo por impedirlo;

- Las actividades recreativas que se realizan en la escuela media se proyectan hacia lo foráneo, desconociendo el valor cultural de lo típicamente patrimonial campesino;

- La literatura cubana que se enseña en la escuela no tiene en cuenta el arsenal de leyendas y mitos campesinos de valor patrimonial. El insuficiente tratamiento a la cultura identitaria campesina hace que los estudiantes no se motiven por esta, que pierdan el interés por investigar lo relacionado con estos valores, los cuales deben estar encaminado a coadyuvar a la conformación del pensamiento histórico y socio-cultural;

- $\quad$ El docente en la planificación de sus actividades descuida las potencialidades educativas que le ofrece la localidad con su patrimonio cultural, las cuales podría insertar en las asignaturas, sin que esto signifique cambios en el currículo. Las potencialidades mayores, y por lo tanto las mayores dificultades, se presentan en asignaturas priorizadas como Historia y Español, extendiéndose a Geografía y, en menor grado, a otras de Ciencias Naturales.

- $\quad$ La identidad cultural, como transversal en los programas de las distintas asignaturas está concebido desde la cultura urbana, con muy pocas valoraciones de los aportes identitarios patrimoniales que tienen como base la cultura campesina.

Respecto a la educación media en Brasil se constata a través de las fuentes publicadas que:

- $\quad$ "Se encontra restrita a projetos isolados, geralmente como experiências piloto com pouca continuidade e pouca profundidade temporal" (HAIGERT, 2003, p. 33).

- En algunas regiones de Brasil se vienen preocupando por el desarrollo y aplicación práctica de la Educación Patrimonial. Los estados de Rio Grande do Sul y Minas Gerais se destacan como los que más han discutido y articulado acciones en este sentido. Por lo menos es lo que se deduce a partir de un exhaustivo análisis bibliográfico.

- Pocos son los ejemplos de acciones concretas de Educación Patrimonial en otros estados, siempre se configuran como acciones aisladas, más que nada por una decisión de algunos municipios.

- $\quad$ Respecto a las fiestas populares y religiosas, no son reconocidos como patrimonio intangible ni por la comunidad no por el estudiantado. Además todo el amplio acervo de conocimientos y saberes no son entendidos como tal. (TEIXEIRA, 2006).

- Los profesores no están preparados para tratar el tema con los alumnos. Ellos mismos no tienen claro los conceptos sobre el tema y cuando los tienen son bastante confusos. Muchos no consiguen diferenciar los conceptos de patrimonio histórico y bien tombado y para muchos de ellos es muy confuso el concepto de patrimonio inmaterial o patrimonio campesino.

- $\quad$ "La concepción de patrimonio no es completamente compartida por los estudiantes. Mencionan muy poco su realidad y sus formas propias de crear cultura. Por todo son discriminados y llevados a creer que 
no producen una cultura digna, no comparten la Historia Oficial, sino como los eternos esclavos. La Historia pertenece a la nación e históricamente ellos han sido excluidos" (TEIXEIRA, 2006, p.139).

En Cuba y en Brasil existe un desarraigo en la cultura patrimonial, especialmente del patrimonio intangible. No puede haber una correcta educación, - entiéndase educación en el sentido amplio, es decir, la formación integral de los adolescentes secundarios,- sin una correcta educación patrimonial que potencie la identidad cultural que es potenciar los valores mismos de la nación. Respecto a los sujetos sociales sin cultura, Freire los consideraba, de forma genérica, como um sujeto "a-histórico, um ser como este não pode comprometer-se; em lugar de relacionar-se com o mundo, o ser imerso nele somente está em contato com ele. Seus contatos não chegam a transformar o mundo, pois deles não resultam produtos significativos, capazes de (inclusive voltando-se sobre ele) marcá-los" (FREIRE, 1981 p. 17). Y es que con una correcta educación patrimonial el sujeto de cultura queda marcado y se convierte de sujeto de cultura en sujeto de identidad cultural.

Para lograr que el sujeto de cultura quede marcado y se convierte de sujeto de cultura en sujeto de identidad cultural es preciso que la escuela juegue un papel decisivo a través del profesor. El profesor debe tener presente que el contacto con el patrimonio cultural es una vía para que el alumno se siente relacionado con su identidad cultural, con sus raíces. En este proceso el estudiante no es un ente pasivo, sino que investiga, descubre, redescubre y participa en la colección y organización del patrimonio cultural. En este medio el estudiante siente un estado emotivo especial, donde la búsqueda del nuevo contenido, a través del patrimonio cultural, se convierte en una necesidad.

Este encuentro no debe ser un simple regreso al pasado, sino el basamento para que el estudiante se sienta parte integrante de la sociedad y capaz de contribuir a su protección y desarrollo; y transformar la sociedad que es también transformar el mundo, como aseveró Freire.

\section{Conclusiones}

La posmodernidad y el proceso globalizador traen consigo el desarraigo cultural y con ello la pérdida de los valores patrimoniales. La UNESCO y muchas instituciones gubernamentales realizan esfuerzos ingentes por conservar esa riqueza cultural heredada históricamente.

La autenticidad en el patrimonio cultural es la esencia de sus valores transcendiendo en el tiempo, y no la originalidad de la obra como fue concebida, ya sea una obra tangible o intangible. La dialéctica del cambio transforma la obra y abre nuevas perspectivas, pero sus atributos deben permancer para garantizar su autenticidad.

Cuba y Brasil difieren en cuanto a la defensa del patrimonio cultural material o natural como una forma de defender la identidad cultural nacional. Cuba posee siete de los quince sitios culturales o naturales de todo el Caribe. Las administraciones municipales en Brasil se desentienden de la preservación patrimonial.

El patrimonio intangible contiene al patrimonio cultural campesino y este es parte inalienable de la identidad cultural campesina. El patrimonio intangible es la conjunción de los aportes de las culturas 
primigenias y de lo nuevo que se ha ido creando a lo largo de los siglos; es el alma de la comunidad, el sentir y el comprender del pueblo a lo largo de generaciones.

Los estudiantes de la enseñanza media en Cuba y Brasil muestran, al igual que sus instituciones un desaliento por la conservación de los valores patrimoniales intangibles. Se expresa este desaliento en sus modos de actuación con la mirada puesta a las culturas extranjerizantes.

Las escuelas, en la enseñanza media, en Cuba y Brasil no aprovechan todo el potencial docente para explotar los conocimiento patrimoniales que potencien una cultura identitaria y a la vez una cultura política de identidad y de defensa.

\section{Bibliografía}

ARGAILLOT, Janice: Cuba y el patrimonio cultural cubano y caribeño desde los principios de la Revolución. https://hal.archives-ouvertes.fr/hal-00941237 2012

ARGAILLOT, Janice: Cuba y el patrimonio cultural cubano y caribeño desde los principios de la revolución. Disponível em: www.red-redial.net/revista/anuario-americanista-europeo/.../211 2013. Acesso em: Agosto de 2019

BALLART, Josep: El patrimonio histórico y arqueológico: valor y uso. Editorial Ariel, S.A. Barcelona. 1997.

BEGOT, Danielle: "Le patrimoine est-il soluble dans le développement durable? Une interrogation à partir de la caraïbe ». En la Caraïbe, un espace pluriel en question. Editado por thierry hartog. Paris: karthala, 2011.

CABEZA, María del Carmen Díaz: Estudios Históoricos. Reflexiones: Tiempos líquidos sobre el Patrimonio Cultural y sus Valores - CDHRP- Agosto 2009 - No 2. Disponível em: www.estudioshistoricos.org/edicion_2/maria_diaz.pdf Acesso em: Agosto de 2019

CÁNOVAS, Elisa Rico: La correspondencia entre la construcción de experiencias auténticas y la interpretación del patrimonio. Reflexiones desde la autenticidad existencial. Disponível em:

www.pasosonline.org/es/articulos/download/file?fid=57.916 Acesso em: Agosto de 2019

CARRERA, Gema: El patrimonio inmaterial o intangible. Disponível em:

www.juntadeandalucia.es/educacion/vscripts/wbi/w/rec/3332.pd. 2018 Acesso em: Agosto de 2019

CARTA DE ATENAS de 1931. Disponível em:

http://www.mcu.es/patrimonio/docs/MC/IPHE/Biblioteca/carta de atenas.pdf. Acesso em Agosto de 2019

CARTA DE BRASILIA: Documento regional del cono sur sobre autenticidad. 1995. Disponível em: portal.iphan.gov.br/uploads/ckfinder/arquivos/Carta\%20Brasilia\%201995.pdf Acesso em Agosto de 2019

CONFERENCIA DE NARA SOBRE AUTENTICIDAD 1994. Disponível em: https://www.culturanorte.pt/.../1994-declaracao de nara sobre autenticidade-icomos

DESANTES REAL, Manuel. Hacia una visión holística del Patrimonio Cultural. Disponível em: https://studylib.es/doc/8091180/completo-en-pdf Acesso em: agosto/2019

FERNÁNDEZ, Antonio J. Sánchez: Patrimonio auténtico, turismo auténtico. influencia de la cultura postmoderna en el concepto de restauración. Disponível em:

https://www.researchgate.net/.../317284120 patrimonio autentico. Acesso em: agosto/2019

FREIRE, Paulo: Educação e Mudança. Rio de Janeiro: Ed. Paz e Terra. 1981.

FUNARI, Paulo A: Os desafios da destruição e conservação do património cultural no brasil. Disponível em: www.ufff.br/maea/files/2009/10/texto1.pdf Acesso em: Agosto de 2019

GONZÁLEZ, Antoni Moreno-Navarro: “Falso histórico o falso arquitectónico, cuestión de identidad", en loggia, nº año i. valencia, universidad politécnica de valencia.

GÚTIEZ, Alejandro Carrión: Plan nacional de salvaguarda del patrimonio cultural inmaterial. Disponível em: www.mecd.gob.es/planes-nacionales/.../08-maquetado-patrimonio Acesso em: Agosto de 2019 
HAIGERT, Cynthia Gindri: Estado da arte sobre educação patrimonial. in: Soares, André 1. r. Educação Patrimonial: relatos e experiências. Santa Maria: Editora UFSM, pp. 33-41. (2003).

UNESCO. La UNESCO y el Patrimonio Inmaterial - portal de la cultura de Disponível em: www.lacult.unesco.org/.../oralidad 11 6-9-la-unesco-y-el-patrim Acesso em: agosto/2019

LAMAS, Raúl Horacio: El patrimonio como oportunidad en la posmodernidad. Disponível em: www.icomos.org.ar/wp- content/uploads/2013/05/lamas.pdf Acesso em: Agosto de 2019

SISCRED. Lineamientos para la Identificación y Manejo del Patrimonio Cultural Inmaterial. Disponível em: siscred.scrd.gov.co/biblioteca/handle/123456789/93 Acesso em: agosto/2019

PÉREZ, Marcelo Tapia: Patrimonio tangible e intangible: aportes al debate del distingo, desde las "prácticas agroculturales" 2012.

PÉREZ, Marcelo Tapia: Patrimonio tangible e intangible: aportes al debate del distingo, desde las "prácticas agroculturales." Disponível em: www.academia.edu/.../patrimonio tangible e intangible aportes... Acesso em: agosto/2019

ROCABADO, Carlos: Patrimonio intangible. Valoración y política cultural: el ejemplo boliviano Cultura oral y Patrimonio Inmaterial: investigación transnacional en el marco de los proyectos Mediterranean Voices y Medins. Disponível em: ccfib.mcu.es/patrimonio/.../PatrimonioCulturalE/.../15-Cultura_or.. Acesso em: agosto/2019

SOLÁ-MORALES, Ignasi: Mies van der rohe: el pabellón de barcelona. barcelona, gustavo gili. 1993.

TEIXEIRA, Simonne: Estudios Pedagógicos XXI, nº 2: 133-145, 2006. Educacion patrimonial: alfabetizacion cultural para la cidadania. Disponível em: www.redalyc.org/pdf/1735/173514131007.pdf Acesso em: agosto/2019

VELASCO, Honorio M: De patrimonios culturales y sus categorías Of cultural heritage and its categories. Disponível em: www.gazeta-antropologia.es/?p=4066 Acesso em: agosto/2019

UNESCO. La UNESCO: DECLARACIÓN DE MÉXICO SOBRE LAS POLÍTICAS CULTURALES Conferencia mundial sobre las políticas culturales México D.F., 26 de julio - 6 de agosto de 1982. Disponível em: www.culturalrights.net/descargas/drets culturals400.pdf Acesso em: agosto/2019

\section{Notas:}

${ }^{1}$ Possui graduação em Filosofia pela Pontifícia Universidade Católica de Campinas (1992), especialização em Economia do Trabalho e Sindicalismo pela Universidade Estadual de Campinas (1995), mestrado em Educação pela Universidade Estadual de Campinas (1998) e doutorado em Educação pela Universidade Estadual de Campinas (2003). Atualmente é professor adjunto da Universidade Federal de São Carlos e Membro de corpo editorial da RECE. Revista Eletrônica de Ciências da Educação. Tem experiência na área de Educação, com ênfase em Fundamentos da Educação. Atuando principalmente nos seguintes temas: educação rural, educação rural- Brasil, escola normal rural, movimento dos trabalhadores rurais sem-terra, ruralismo pedagógico. ORCID: Email: lbezerra@ufscar.br

2 Universidade de Holguin - Cuba - ORCID: Email: juancarlosrodriguezcruz2002@gmail.com

${ }^{3}$ La situación de este país es bastante excepcional, primero en comparación con el resto del Caribe, ya que solo posee siete de los quince sitios, culturales o naturales, distinguidos por la UNESCO para esta región del mundo.], pero también por la variedad de lo que se ha considerado digno de ingresar a la memoria colectiva del planeta. Los siete sitios arquitectónicos seleccionados (la ciudad vieja de La Habana y sus fortificaciones, la ciudadela de Santiago, los restos de las plantaciones de café de la región de Santiago, Trinidad y el valle azucarero de Los Ingenios, el valle de Viñales, los antiguos corazones de Cienfuegos y Camagüey ...) son además dos propiedades "naturales" (los parques de Desembarco de Granma y A. Humboldt), y más notables, porque es el único ejemplo en el Caribe para el patrimonio intangible, el Certificación de la Tumba francesa de Santiago, una asociación de canciones y bailes afrocubanos.

${ }^{4}$ La distancia entre las autoridades y el pueblo es la misma de aquella entre la sociedad civil y el pasado, debido a la falta de información, aunque los habitantes de las ciudades coloniales dependen del turismo para su propia supervivencia. ¿Quiénes son los mayores enemigos de la preservación de esas ciudades coloniales? En primer lugar, la propia administración municipal, no afectada por los problemas sociales e ignorantes de las cuestiones culturales en general, pero a veces los habitantes también, inconscientes de la importancia de los monumentos, contribuyen a la deformación del marco urbano. Nuevas ventanas, antenas parabólicas, garajes, techos y casas enteras bastan para transformar una ciudad colonial en una ciudad moderna, una mera sombra de una antigua ciudad colonial, como es el caso de tantas de ellas. 